\title{
Exogenous miR-29B Delivery Through a Hyaluronan-Based Injectable System Yields Functional Maintenance of the Infarcted Myocardium
}

\author{
Michael G. Monaghan, PhD, ${ }^{1-3}$ Monika Holeiter, PhD, ${ }^{1,2}$ Eva Brauchle, PhD, ${ }^{1,2}$ Shannon L. Layland, MBA,,2 \\ Yan Lu, PhD, Arjun Deb, MD, Abhay Pandit, PhD, Ali Nsair, MD, and Katja Schenke-Layland, PhD, MSc ${ }^{1,2,4}$
}

Myocardial infarction (MI) results in debilitating remodeling of the myocardial extracellular matrix (ECM). In this proof-of-principle study it was sought to modulate this aggressive remodeling by injecting a hyaluronic acid-based reservoir delivering exogenous microRNA-29B (miR-29B). This proof-of-principal study was executed whereby myocardial ischemia/reperfusion was performed on C57BL/6 mice for 45 min after which five $10 \mu \mathrm{L}$ boluses of a hydrogel composed of thiolated hyaluronic acid cross-linked with poly (ethylene glycol) diacrylate, containing exogenous miR-29B as an active therapy, were injected into the border zone of the infarcted myocardium. Following surgery, the myocardial function of the animals was monitored up to 5 weeks. Delivering miR-29B locally using an injectable hyaluronan-based hydrogel resulted in the maintenance of myocardial function at 2 and 5 weeks following MI in this proof-of-principle study. In addition, while animals treated with the control of a nontargeting miR delivered using the hyaluronan-based hydrogel had a significant deterioration of myocardial function, those treated with miR-29B did not. Histological analysis revealed a significantly decreased presence of elastin and significantly less immature/newly deposited collagen fibers at the border zone of the infarct. Increased vascularity of the myocardial scar was also detected and Raman microspectroscopy discovered significantly altered ECM-specific biochemical signals at the border zone of the infarct. This preclinical proof-of-principle study demonstrates that an injectable hyaluronic acid hydrogel system could be capable of delivering miR-29B toward maintaining cardiac function following MI. In addition, Raman microspectroscopy revealed subtle, yet significant changes in ECM organization and maturity. These findings have great potential with regard to using injectable biomaterials as a local treatment for ischemic tissue and exogenous miRs to modulate tissue remodeling.

Keywords: heart, collagen, elastin, cardiac infarct

\section{Introduction}

W ORLDWIDE, CORONARY ARTERY DISEASE is the single most frequent cause of death, which is increasing with 7.4 million people dying each year, accounting for $13.2 \%$ of all deaths. ${ }^{1}$ It is estimated that every sixth man and every seventh woman will die from myocardial infarction (MI) in Europe. ${ }^{2}$ Despite significant advances in post-MI therapies, surviving patients often develop chronic heart failure, a severe condition with high mortality and morbidity rates, affecting more than 24 million people worldwide. ${ }^{2}$ The adult mammalian heart has negligible regenerative

\footnotetext{
${ }^{1}$ Department of Women's Health, Research Institute for Women's Health, Eberhard Karls University, Tübingen, Germany.

${ }^{2}$ Department of Cell and Tissue Engineering, Fraunhofer Institute for Interfacial Engineering and Biotechnology (IGB), Stuttgart, Germany.

${ }^{3}$ Department of Mechanical and Manufacturing Engineering, School of Engineering, Trinity College Dublin, the University of Dublin, Dublin, Ireland.

${ }^{4}$ Department of Medicine/Cardiology, Cardiovascular Research Laboratories (CVRL), University of California (UCLA), Los Angeles, California.

${ }^{5}$ Centre for Research in Medical Devices (CÚRAM), National University of Ireland, Galway, Ireland.

(c) Michael G. Monaghan et al. 2018; Published by Mary Ann Liebert, Inc. This article is available under the Creative Commons License CC-BY-NC (http://creativecommons.org/licenses/by-nc/4.0). This license permits non-commercial use, distribution and reproduction in any medium, provided the original work is properly cited. Permission only needs to be obtained for commercial use and can be done via RightsLink.
} 
capability; therefore, a highly aggressive alteration of cardiac tissue compensates the loss of cardiomyocytes following MI leading to fibrotic scar formation and debilitating remodeling, inevitably resulting in a loss of contractile myocardium. ${ }^{3}$ An increased synthesis of fibrillar type I and type III collagens becomes morphologically evident at week 1 post-MI and their organized assembly in the form of scar tissue is evident at week 2 , which continues to accumulate over 8 weeks. ${ }^{4}$ This excessive accumulation of extracellular matrix (ECM) proteins in the interstitial and perivascular regions of the myocardium during cardiac fibrosis is a hallmark of maladaptive hypertrophy and heart failure. ${ }^{3}$

A disruptive imbalance of microRNAs (miRs) occurs within the myocardium that is intimately coordinated with the heart's response to MI. ${ }^{5}$ miRs are a vital and evolutionary component of gene regulation and serve to posttranscriptionally silence mRNAs through translation inhibition and degradation. ${ }^{6}$ miR-29B has been specifically associated with the regulation of fibrosis in a number of tissues, including renal, bone, ${ }^{7}$ pulmonary, hepatic, ${ }^{8}$ and cardiac tissue, ${ }^{9}$ and has also been elucidated to play a significant role in ECM remodeling, possessing a significant relationship with collagen production. ${ }^{9,10}$ It has been collectively shown that the miR-29 family members target at least 16 genes related to ECM, which encode for several key proteins involved in physiological or pathological formation of ECM, including a large number of collagen isoforms, laminin $\gamma$, fibrillin 1, elastin, MMP 2, and integrin $\beta 1 .^{9,11}$ This is unique to the miR-29 family as no other miR is predicted to target more than 11 of the 20-collagen genes. In cultured cardiac fibroblasts, miR-29 has been downregulated after TGF- $\beta_{1}$ stimulation, suggesting that the decrease in miR-29 in the pathological remodeling of the heart is mediated by TGF- $\beta_{1} .{ }^{9}$ Van Rooij et al. were the first to show that the miR-29 family directly targets a multitude of ECM genes such as collagens type I and III, elastin, and fibrillins. ${ }^{9}$ The reported downregulation of miR-29 in several cardiac pathologies suggests that this loss may actually contribute to the development of cardiac fibrosis by relieving its repression on ECM gene expression. Knockdown of miR-29B by antisense oligonucleotides (antagomiRs), designed to block miR-29B in the healthy mouse heart, has resulted in increased expression of ECM genes at the mRNA level, but it is unclear whether this was sufficient to induce excessive fibrosis. ${ }^{9}$ Using miRs as therapeutics has recently gathered interest as miR mimics and antagomiRs can be easily produced and synthesized, and the mechanism by which they function requires cellular entry rather than nuclear insertion (as is the case for plasmid DNA). ${ }^{12,13}$

This proof-of-principle study hypothesizes that the delivery of exogenous miR-29B following MI will contribute toward an improved functional recovery of the myocardium through modification of the deposited ECM. To ensure a localized delivery, minimizing off-target effects on other organs, we aimed to deliver miR-29B at the border zone of the infarct using an injectable hydrogel based on thiolmodified hyaluronan, cross-linked with poly (ethylene-glycol) diacrylate. In addition, Raman microspectroscopy was used as a complimentary tool to detect changes in ECM organization and maturity not detectable by marker-depended conventional methods.

\section{Materials and Methods}

\section{Hyaluronan-based hydrogel/miR fabrication}

Glycosan HyStem ${ }^{\circledR}$ (BioTime, Inc.), a thiolated hyaluronanbased hydrogel cross-linked using thiol-reactive poly(ethylene glycol) diacrylate, was diluted with phosphate-buffered saline (PBS; 13:2 ratio, PBS:HyStem). miR-29B and miR239B mimics (negative control, which has been confirmed to have minimal sequence identity with miRs in human, mouse, and rat) were obtained from Dharmacon ${ }^{\mathrm{TM}}$ with the sequences for:

cel-miR-239B: 5'-uuuguacuucggcuaaggugcug-3' (nontargeting control)

mur-miR-29B: 5'-uagcaccauuugaaaucaguguu-3'

and reconstituted in the PBS and mixed with the hydrogel solution (13:2) to achieve a final miR concentration of $140 \mu \mathrm{M}$ of miR mimics (Fig. 1). Hydrogel solutions were prepared directly before injection to prevent premature gelation, whereby at room temperature gelation occurs within 20 min.

\section{Ischemia/reperfusion studies}

Animal care and surgeries were performed in compliance with the University of California Los Angeles Animal Research Committee under protocols 2011-042 and 2013-057. Ischemia/reperfusion was conducted on C57BL/6 mice $(20 \pm 1.2 \mathrm{~g}$ body weight) through ligation of the left anterior descending (LAD) artery as previously described. ${ }^{14}$ Briefly, three animals per group were anesthetized with isoflurane, and a left thoracotomy under mechanical ventilation using a volume cycled rodent ventilator (Harvard MiniVent) was performed. The LAD coronary artery was ligated intramurally, $2 \mathrm{~mm}$ from its origin using a 9-0 nylon suture. A small plastic tube (PE-10) was placed between the ligated vessel, and the node was left tightened against the LAD for $45 \mathrm{~min}$ whereby ischemia was verified by subsequent blanching of the myocardium. Reperfusion was accomplished by cutting the knot on the PE-10 tube and confirmed by hyperemia of the previously pale ischemic region.

Animals received serial injections of $10 \mu \mathrm{L}$ volumes of hydrogel solution (containing miR-29B or the control of miR-239B) into five sites at the infarction border zone (total $=50 \mu \mathrm{L}$ ), identified by regional blanching of the myocardium. As controls, $10 \mu \mathrm{L}$ volumes of hydrogel solution (containing no miRs) and $10 \mu \mathrm{L}$ volumes of saline were injected into five sites at the infarction border zone (total $=50 \mu \mathrm{L}$ ). The veterinary surgeon administered the treatments in a blinded manner. After confirmation of no internal bleeding, the chest was closed in two layers as follows: first the ribs (inner layer) were closed with 6-0 coated Vicryl ${ }^{\circledR}$ sutures in an interrupted pattern, and the skin was closed using 6-0 nylon or silk sutures in a subcuticular manner. The anesthesia was removed, and the animals were allowed to recover respiration before the endotracheal tube was removed. All mice received $2.5 \mathrm{mg} / \mathrm{kg}$ Banamine postsurgery.

\section{Echocardiography}

Mice were assessed echocardiographically presurgery to establish a pretreatment baseline and again at the time points of 2 days, 2 weeks, and 5 weeks postsurgery to track cardiac function using a high-frequency ultrasound 
A

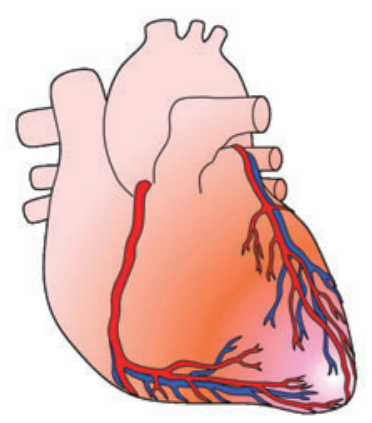

Ischemia/Reperfusion

D

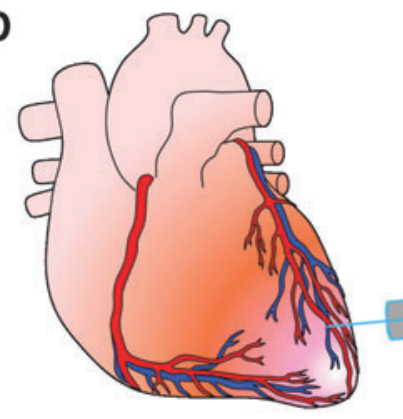

Intramyocardial Injection
B

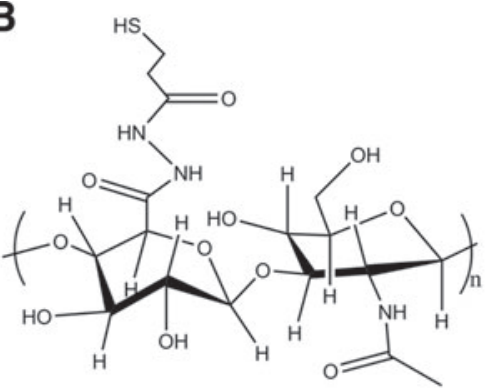

Thiol Modified Hyaluronic Acid
C

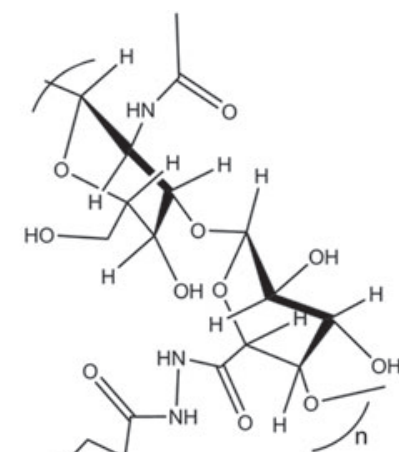

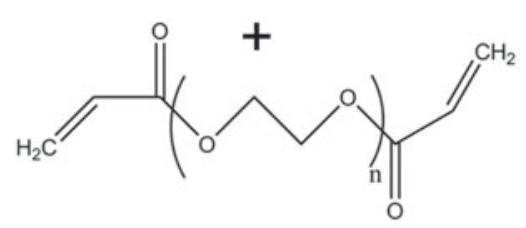

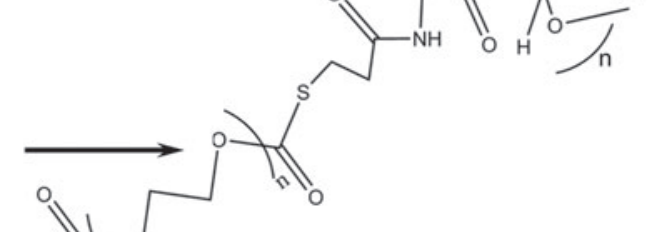

PEGDA

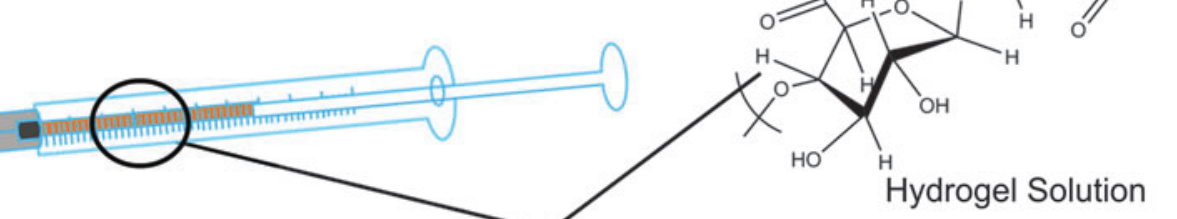

Hydrogel Solution

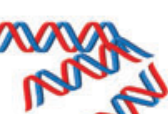

microRNA Mimics

E
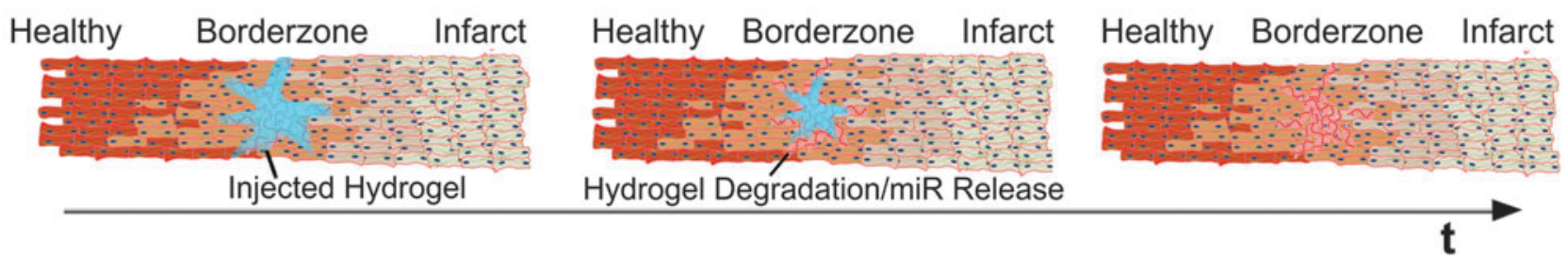

FIG. 1. (A) Schematic of the MI model and hyaluronan-based delivery system used in this study. (B) Thiol-modified hyaluronic acid was reacted with PEGDA to form a hydrogel solution. (C) miR mimics were mixed with the hydrogel solution and prepared in an insulin syringe. (D) The hydrogel/miR mimic mixture was injected into the border zone of infarcted myocardium following ischemia/reperfusion injury. (E) Hypothesized behavior and release kinetics of miRs from the injected hydrogel over time. As the hydrogel (blue) is injected into the border zone of the infracted myocardium, cells and ECM present at this location become exposed to the injected treatment. Over time ( $t$ ), it is possible that degradation of the hydrogel in combination with release of miR mimics from the hydrogel results in the highest concentration of miR mimics being present close to the site of injection at the border zone with reduced presence further away from the injection site. MI, myocardial infarction; miR, microRNA; PEGDA, poly (ethylene glycol) diacrylate.

system (Vevo ${ }^{\circledR} 2100$; FujiFilm VisualSonics, Inc.). This was performed by the same veterinary surgeon who administered the treatments, who was therefore unaware of the treatment each animal received. Image acquisition was performed at a high frame rate ( $>200$ frames/s) to ensure an adequate quality of the images and for at least three cardiac cycles per loop where breathing artifacts were absent or minimized. Animals which exhibited incomplete ischemia at day 2 were removed from the study.

\section{Histology and immunofluorescence staining}

Animals were sacrificed at 5 weeks following MI. Following morphometric planimetry, explanted hearts were fixed for $12 \mathrm{~h}$ in $10 \%$ formalin, subjected to gradual serial dehydration, and embedded in paraffin. Entire hearts were then sectioned into $3 \mu \mathrm{m}$ thick sections. For general morphology serial sections (every tenth section) were deparaffinized in xylene, rehydrated through graded ethanol 
washes $(100-70 \% \mathrm{v} / \mathrm{v})$ to water, and stained in $0.1 \%$ fast green ( $\mathrm{pH} \mathrm{7,} \mathrm{Fast} \mathrm{Green} \mathrm{FCF;} \mathrm{Sigma} \mathrm{Aldrich)} \mathrm{for} 30 \mathrm{~min}$ followed by staining in $0.1 \%$ Sirius red in saturated picric acid (picrosirius red stain) for $1 \mathrm{~h}$. Following this, the sections were rapidly dehydrated through graded ethanol washes $(70-100 \% \mathrm{v} / \mathrm{v})$ and mounted. Bright field and orthogonal images were taken at $63 \times$ and $40 \times$ magnification with a light microscope Observer.Z1 (Carl Zeiss GmbH). After identification of the infarcted tissue sections, the area of the fibrous infarcted myocardium was calculated as the percentage of the picrosirius red-stained collagen in the myocardium per total area of tissue (picrosirius red- and fast green-stained tissue) using bright field images. Features such as the cardiac valves were omitted from these calculations. Areas were calculated using threshold analyses using ImageJ (freely available from www.nih.org).

For immunofluorescence staining, sections were deparaffinized in xylene, rehydrated through graded ethanol washes $(100-70 \% \mathrm{v} / \mathrm{v})$ to water, and heat mediated antigen retrieval was performed using a microwaveable pressure cooker as previously described. ${ }^{15}$ The following primary antibodies and dilutions were used for immunohistological staining: cardiac troponin T (cTnT; Sigma Aldrich, HPA015774, 1:3000), platelet endothelial cell adhesion molecule-1 (CD31; Dianova GmbH, DIA-310, 1:20), FITC-conjugated alpha smooth muscle actin (Sigma Aldrich; F3777, 1:500), collagen type I (Acris Antibodies GmbH; R1038, 1:75), lumican (Abcam Plc; ab168348, 1:500), and fibulin 5 (a kind donation from Prof. D. Reinhardt, ${ }^{16}$ 1:500). In all cases, isotype controls were included using only the antibody dilution buffer. The secondary antibodies, Alexa Fluor ${ }^{\circledR}$ (Invitrogen ${ }^{\mathrm{TM}} /$ Thermo Fisher Scientific) goat anti-rabbit (594), goat anti-rat (647), and goat anti-rabbit (488), were used for immunofluorescence staining: all at a dilution of 1:250. After secondary antibody incubation, all sections for immunofluorescence were exposed to a 4',6-diamidino-2-phenylindole (DAPI) solution for $10 \mathrm{~min}$ followed by mounting using ProLong ${ }^{\circledR}$ Gold antifade mounting medium (Molecular Probes, Life Technologies). A minimum of 10 tissue sections were prepared during each staining procedure for imaging analysis.

\section{Image analysis}

Blood vessel density was quantified using pictures from immunofluorescence staining of CD31, cTnT, and $\alpha$ SMA. Measurements were facilitated using ImageJ. The blood vessel density was calculated using the formula:

$$
\text { blood vessel density }=\frac{\text { number of blood vessels }}{\text { scar area }}
$$

Ages of immunofluorescence-stained sections utilizing antibodies against lumican, elastin, collagen type I, and fibulin 5 were analyzed and quantified through the calculation of the gray value intensities (GVI) using ImageJ. The scar region was circumscribed in each image and the GVI within this selected area measured. This was easily discernable using cTnT staining (where present) and also autofluorescence emanating from cardiomyocytes. GVI were then calculated using the measure function of ImageJ, which provides the mean GVI within the selected area.

\section{Directionality of the collagen fibers}

Directionality analysis was performed on the birefringence images to observe the percentage of alignment of collagen fibers using a directionality plugin based on a Fast Fourier Transform analysis with ImageJ. In detail, collagen tissue shows a better alignment when arranged under strain. As the tissue's birefringence is along the direction of strain, it can be estimated as a linear retarder whose fast axis is aligned in the direction of strain. ${ }^{17}$

\section{Raman microspectroscopy}

Paraffin-embedded tissue sections (each $10 \mu \mathrm{m}$ thick) were deparaffinized and analyzed using Raman microspectroscopy. A custom-built Raman microspectroscopy system was used for all measurements as previously described. ${ }^{18}$ Using an automated stage, spectra were automatically acquired from 35 randomly selected points throughout the tissue. This was performed in triplicates for every sample. A total acquisition time of $100 \mathrm{~s}$ was used for each spectrum. Raman spectra were acquired in the wave number range of $0-2000 \mathrm{~cm}^{-1}$ and recorded using the Andor software package (Andor iDus). All measurements were performed from specimens mounted on glass slides. The glass background signal was subtracted from the samples signal using the OPUS ${ }^{\circledR}$ software 4.2 (Bruker Optik $\mathrm{GmbH}$ ). In addition, OPUS software 4.2 was used to segment the Raman spectra into the $400-1800 \mathrm{~cm}^{-1}$ spectral region, whereby baseline correction and vector normalization were performed. All samples were investigated at two regions of the myocardial scar; namely, the center of the infarct and the transition zone between the fibrotic infarct and healthy myocardium (border zone).

\section{Statistical analysis}

Results are depicted throughout as means \pm standard deviation. Statistical analysis was performed using Prism ${ }^{\mathrm{TM}}$ (GraphPad Software). Student's $t$-test or one-way ANOVA was performed where appropriate to determine statistically significant differences among the miR-29B treatment, the control miR-239B treatment, and the additional controls of hydrogel with no miRs and saline alone injections. Statistical significance was defined at $p<0.05$.

\section{Results}

\section{Delivering miR-29B through an injectable hydrogel scaffold improves functional recovery following MI}

Delivering miR-29B intramyocardially through a hyaluronanbased scaffold at the border zone yielded a significantly improved myocardial function at the follow-on time points of this proof-of-principle MI study (Fig. 2) compared with the treatment of the nontargeting miR-239B control. Specifically, mice treated with miR-29B had a significantly higher ejection fraction compared with mice treated with miR-239B, at 2 and 5 weeks post-MI (nontargeting mammalian control) (Fig. $2 \mathrm{~A}, 51.38 \% \pm 3.19 \%$ vs. $43.70 \% \pm 0.67 \%$ and $52.02 \% \pm$ $4.14 \%$ vs. $42.82 \% \pm 3.23 \%$, respectively; $p<0.05)$. A significant improvement was also observed at 5 weeks in the assessment of fractional shortening (Table 1, 25.58\% $\pm 3.02 \%$ vs. $20.37 \% \pm 1.72 \% ; p<0.05)$ and fractional area change (Table $1,45 \% \pm 6.93 \%$ vs. $30 \% \pm 1 \% ; p<0.05$ ). Moreover, 

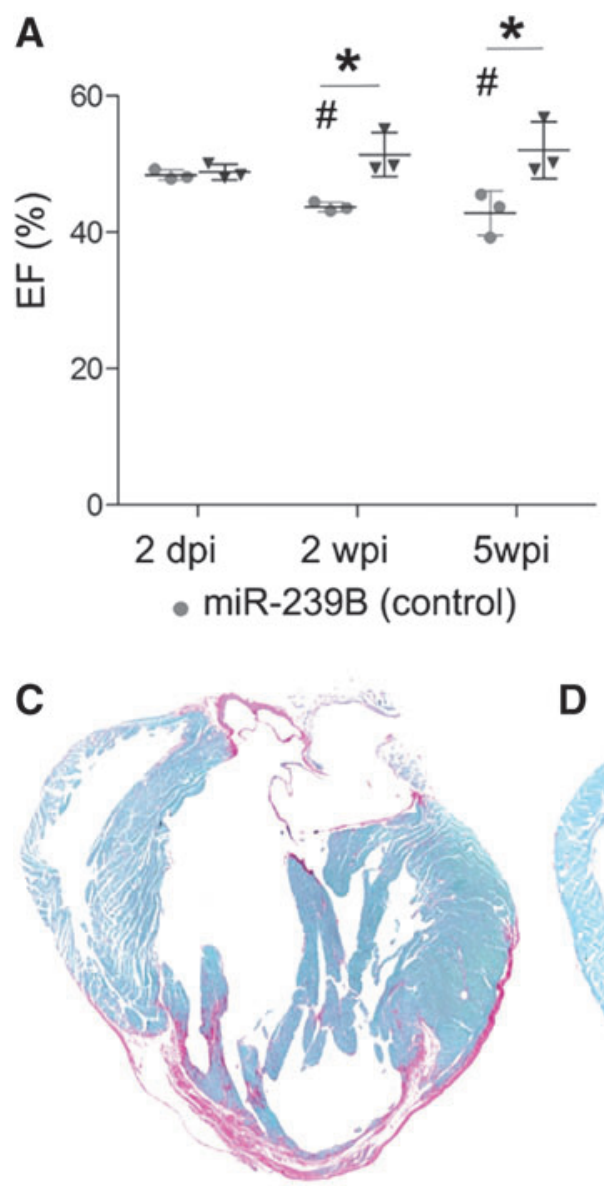

miR-239B (control)

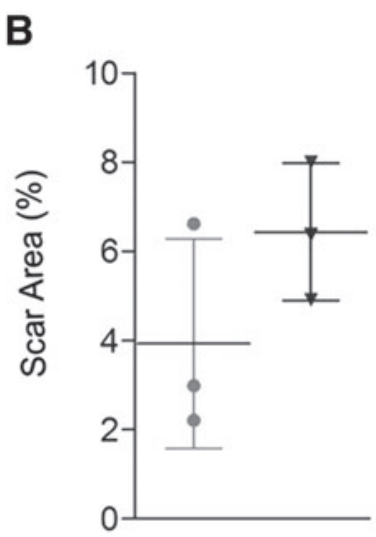

$\checkmark$ miR-29B

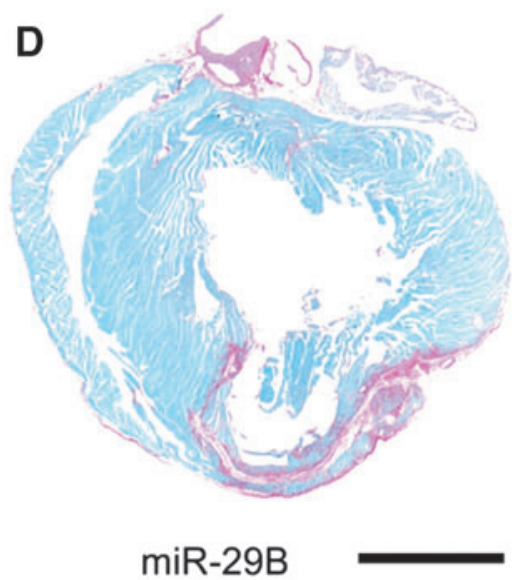

FIG. 2. (A) Morphometric data at 2 dpi and 2 and 5 wpi with treatment of miR-239B (control) or miR-29B. Asterisk (*) indicates a statistically significant improvement in EF. \# Indicates a statistically significant deterioration in the cardiac function of animals treated with miR-239B when compared to the initial measurement at 2 days post infarction $(p<0.05)$. (B) Quantification of scar area as a percentage of the total tissue cross section. No significant difference was detected between miR-239B and miR-29B at 5 weeks post ischemia. Picrosirius red/fast green-stained sections of hearts treated with (C) miR-239B and (D) miR-29B. Scale bar: $2 \mathrm{~mm}$. Red staining in the ventricular tissue represents fibrotic ischemic scar tissue at 5 weeks. dpi, days postinfarction; EF, ejection fraction; wpi, weeks postinfarction. while the ejection fraction of the mice treated with miR239B significantly deteriorated at 2 and 5 weeks post-MI compared to the ejection fraction at 2 days post-MI, mice treated with miR-29B had no significant deterioration in ejection fraction and exhibited a slight trend toward an improved ejection fraction over time. Infarcts treated with hyaluronan-based hydrogels containing no miRs had similar ejection fractions to those treated with hydrogels con- taining the nontargeting miR-239B control (Supplementary Fig. S1; Supplementary Data are available online at www .liebertpub.com/tea). This is significant, in that it highlights the functional specificity of miR-29B and the reliability of the nontargeting miR-239B as a control. In addition, mice treated with just saline injections had significantly reduced ejection fractions (Supplementary Fig. S1) compared with any of the hyaluronan-based hydrogel treatments (with or

Table 1. Morphological Data Obtained from Echocardiographic Measurements From Animals Treated With miR-29B AND MiR-239B (CONTROL)

\begin{tabular}{llcccc}
\hline & & Preinfarction & 2 dpi & 2 wpi & 5 wpi \\
\hline Fractional shortening $(\%)$ & miR-29B & $59.7 \pm 5.5$ & $23.7 \pm 0.8$ & $25.2 \pm 2.2$ & $25.6 \pm 3^{\mathrm{a}}$ \\
& miR-239B & $67.3 \pm 4.4$ & $23.3 \pm 0.7$ & $20.7 \pm 0.7$ & $20.4 \pm 1.7$ \\
Fractional area change $(\%)$ & miR-29B & $57.7 \pm 2.5$ & $37 \pm 4.4$ & $43.3 \pm 5.1$ & $45 \pm 6.9^{\mathrm{a}}$ \\
& miR-239B & $64 \pm 6.7$ & $33 \pm 8$ & $31.3 \pm 6.7$ & $30 \pm 1$ \\
Heart rate $(\mathrm{BPM})$ & miR-29B & $563.7 \pm 49.1$ & $564 . \pm 29.2$ & $553 . \pm 93.9$ & $535.2 \pm 36.3$ \\
& miR-239B & $569.8 \pm 29.9$ & $551.5 \pm 43.3$ & $594.3 \pm 43.3$ & $537.2 \pm 23.6$ \\
Heart mass $(\mathrm{mg})$ & miR-29B & $43.5 \pm 6.4$ & $36 \pm 6.3$ & $35.7 \pm 3.7$ & $36.8 \pm 12.3$ \\
& miR-239B & $46.8 \pm 8.5$ & $32.59 \pm 8.4$ & $37.1 \pm 15.6$ & $47.4 \pm 17.6$ \\
End systolic volume $(\mu \mathrm{L})$ & miR-29B & $2.2 \pm 1.2$ & $17.3 \pm 3.02$ & $14.6 \pm 3.9$ & $15.4 \pm 7.2$ \\
& miR-239B & $1.3 \pm 0.6$ & $15.6 \pm 5.3$ & $21 . \pm 11.5$ & $22.8 \pm 6.6$ \\
End diastolic volume $(\mu \mathrm{L})$ & miR-29B & $21.5 \pm 4.3$ & $33 . \pm 6.5$ & $30.4 \pm 10.4$ & $33.1 \pm 18.5$ \\
& miR-239B & $21.2 \pm 4.5$ & $30.3 \pm 10.3$ & $37.5 \pm 20.8$ & $39.7 \pm 9.8$ \\
\hline
\end{tabular}

\footnotetext{
${ }^{\mathrm{a}}$ Indicates a statistically significant improvement in fractional shorting and fractional area change with treatment of miR-29B when compared with the control treatment of miR-239B at the time points indicated $(p<0.05)$.

BPM, beats per minute; dpi, days postinfarction; wpi, weeks postinfarction.
} 
without miRs), which demonstrate the therapeutic benefit of injecting ECM-based biomaterials into the infarcted myocardium. Based on these findings, we sought to elucidate the underlying reason as to why miR-29B was generating the most beneficial outcome. For this reason, we focused the histological evaluations on infarcts treated with hyaluronanbased hydrogels containing miR-29B and the nontargeting miR control (miR-239B).

\section{miR-29B delivery elicits a significantly altered maturation of collagen fibers and reduced production of elastin at the border zone of the infarcted myocardium}

We next sought to determine the source of the functional recovery of animals treated with miR-29B. We therefore investigated the size of the remodeled fibrotic myocardium (Fig. 2C, D). Interestingly, using stereological approaches, we detected no significant difference between the size of myocardial scars in the miR-239B- and miR-29B-treated mice (Fig. 2B). Considering the impact of the scar on cardiac function and yet not detecting any significant difference in the size of the scar between treatments, we decided to investigate deeper the quality of the resulting scar regarding its orientation and ECM composition. With this in mind, we viewed picrosirius/fast green-stained tissue sections, with particular focus on the center and border zone of the infarct under orthogonal light (Fig. 3A). Collagen fibers are birefringent, a characteristic that is enhanced by picrosirius staining, and it is widely reported that thicker more mature collagen fibers (namely type I collagen fibers) polarize using this method toward a stronger red/orange birefringence and thinner fibers toward a weaker green birefringence. ${ }^{19,20}$ The intensely positive birefringence of collagen is caused by the superimposed right-handed superhelix of the left handed helix consisting of the three polypeptide chains, yielding a consequent alignment of the amino acid chains approximately parallel to the molecular axis. ${ }^{19}$ According to Hirshberg, ${ }^{21}$ greenish yellow collagen fibers can represent procollagen, intermediate, or pathological collagen fibers. Quantification of the presence of mature (red/orange) and immature (green) fibers revealed no significant difference between the infarcts treated with miR-29B or miR-239B (Fig. 3B). Remarkably, however, we detected a statistically significant difference in the proportion of mature/immature fibers in the border zone of the infarcts treated with miR-29B compared with miR239B. Namely, the border zone of the infarcts treated with miR-29B had increased mature fibers and less immature fibers compared with miR-239B-treated infarcts (Fig. 3B). Investigating the directionality of these fibers however revealed no significant difference in fiber orientation at either the border zone or center of the infarcts when comparing miR-29B-treated to miR-239B-treated hearts (Fig. 3C). As picrosirius red is a general collagen stain, we then performed immunofluorescence staining for collagen type I (Fig. 4A), yet found no significant difference in its quantity (determined by assessing GVI, Fig. 4B) within the infarct, at the border zone of the infarct, or the interstitium of the remote myocardium. We investigated several other ECM components present in fibrillogenesis, but found no
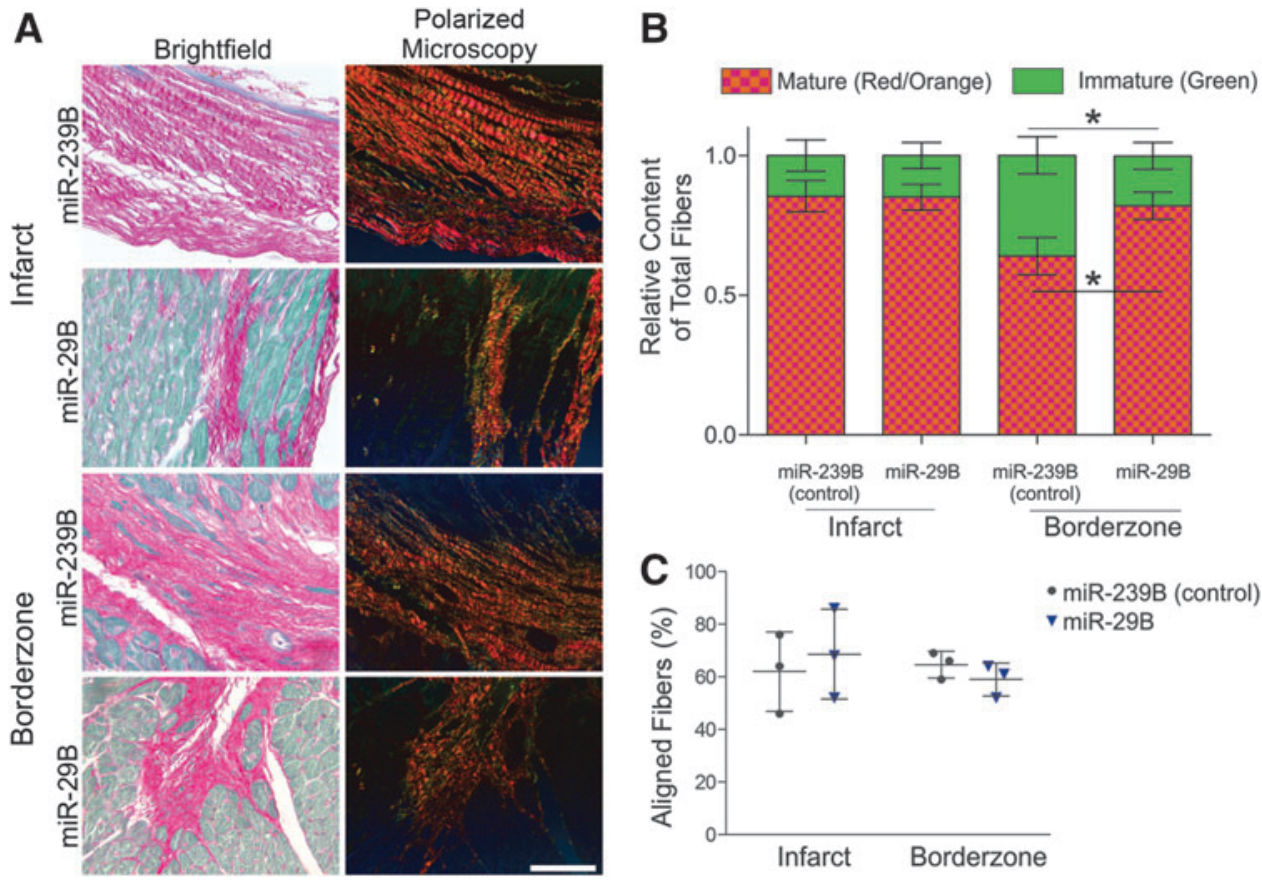

FIG. 3. Analysis of scar fiber orientation and quality. (A) Bright field microscopy of picrosirius red/fast green-stained sections in high magnification and with microscopy of the same regions of interest under orthogonal polarized light to enhance birefringence of collagen fibers. Scale bar: $100 \mu \mathrm{m}$. (B) Quantification of birefringent fibers using color threshold segmentation for mature fibers (red/orange) and immature fibers (green). A statistically significant increased proportion of immature fibers and decreased proportion of mature fibers were revealed at the border zone of infarcts treated with the miR239B (control) compared with infarcts treated with miR-29B $\left({ }^{*} p<0.05\right)$. (C) Quantification of fiber orientation with respect to the center and the border zone of the infarct. No statistically significant difference was detected in the percentage of fibers orientated to the myocardial wall with miR-29B-treated infarcts compared with miR-239B-treated infarcts. 


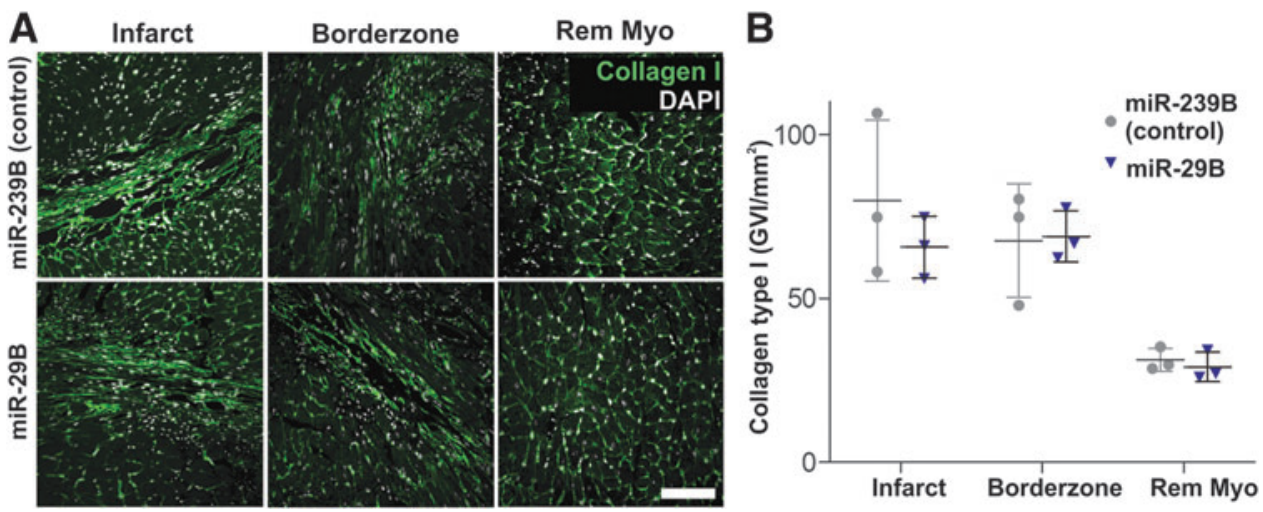

FIG. 4. (A) Immunofluorescence micrographs of cardiac sections stained for collagen type I (green) with nuclear counterstaining using DAPI (white) and imaged within the infarct, at the border zone and at the remote myocardium (Rem Myo). Scale bar: $100 \mu \mathrm{m}$. (B) The graph presents quantification of GVI from the collagen type I staining. No statistically significant difference was detected between the collagen expression of miR-29B samples compared with miR-239B (control samples). DAPI, 4',6-diamidino-2-phenylindole; GVI, gray value intensities.

significant difference in the expression of these proteins after GVI quantification (lumican and fibulin 5, Supplementary Fig. S2).

As miR-29B is a known suppressor of elastin production, ${ }^{9}$ we sought to evaluate the elastin presence of the myocardial scar at both the center and border zone of the infarct (Fig. 5A). Much like findings from the quantification of polarized fibers, we detected a statistically significant difference at the border zone of the infarcted myocardium with the treatment of miR29B (Fig. 5B). Herein, we detected a significant reduction in elastin expression (through quantification of GVI of elastin immunofluorescence staining) in miR-29B-treated hearts compared with miR-239B (control)-treated hearts (Fig. 5A-C, $2.983 \pm 0.6764$ vs. $4.396 \pm 0.3207 \mathrm{GVI} / \mathrm{mm}^{2}$ ). No significant difference in elastin expression was detected between the centers of the infarcts of these treatments. In addition, we investigated the vascularity of the remodeled myocardial scar by evaluation of blood vessel density within the scar tissue (Fig. 5C). Although no statistically significant difference was detected, there was a strong trend toward increased blood vessel density at both the
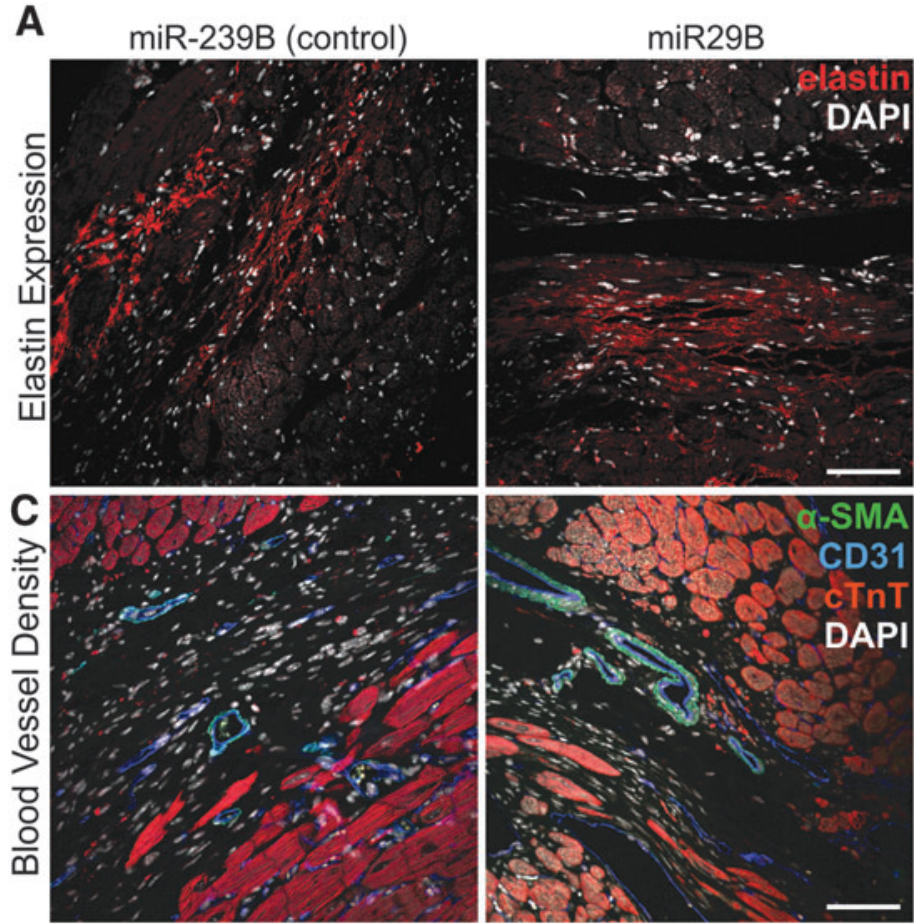

B
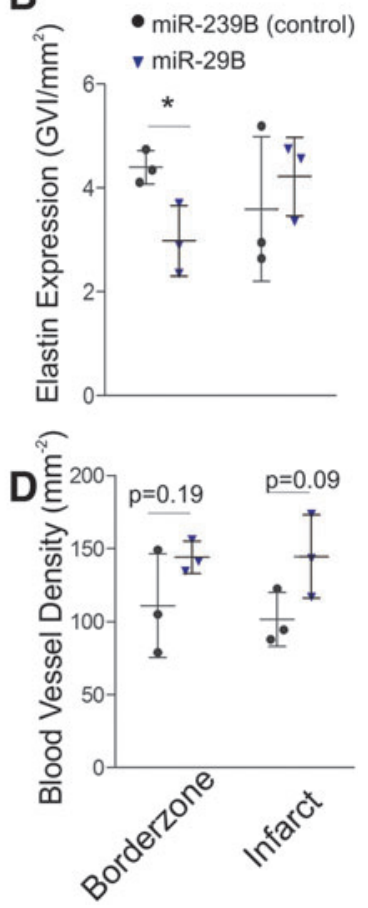

FIG. 5. (A) Immunostaining and (B) quantification of elastin expression in infarcts treated with miR-29B and miR-239B. Scale bar: $100 \mu \mathrm{m}$. A significantly decreased presence of elastin was revealed in the border zone of infarcts treated with the miR-29B compared with infarcts treated with miR-29B $(* p<0.05)$. (C) Immunostaining for detection of blood vessel density within myocardial scars and (D) quantification of vessel density within myocardial scars. A strong trend was detected toward increased vascularity at both the border zone $(p=0.19)$ and center of the infarcts $(p=0.09)$ of hearts treated with miR-29B compared with control treatments (miR-239B). 


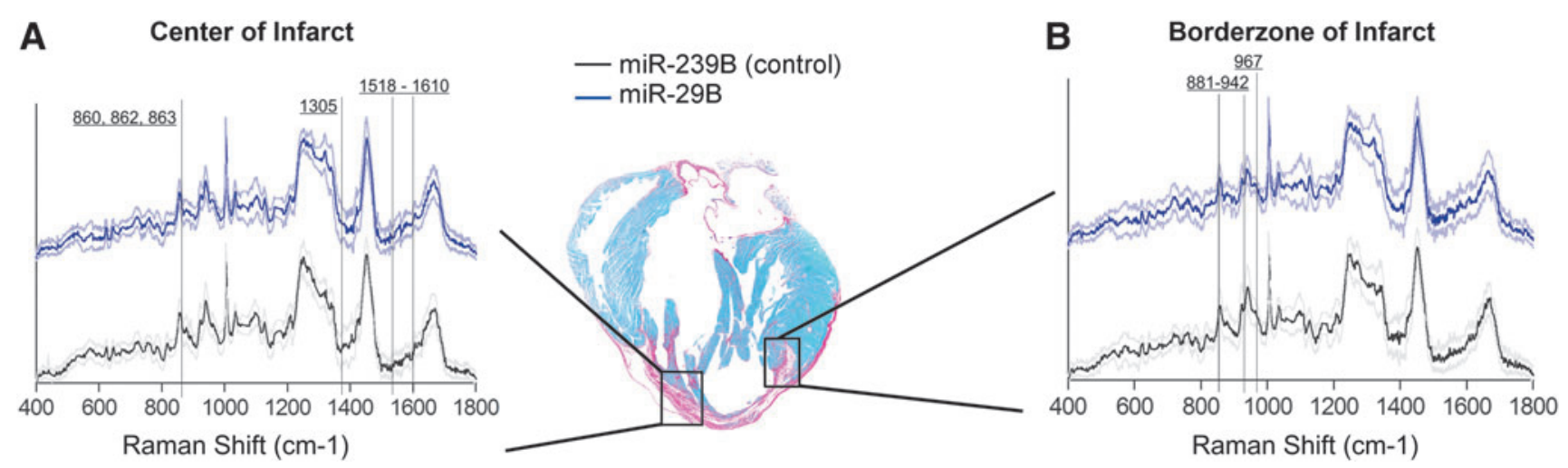

FIG. 6. Raman spectra acquired at the center (A) and the border zone of the infarct (B) at 5 weeks post-MI in miR-239B-and miR-29B-treated samples. Vertical lines indicate spectra of interest, which revealed spectral differences between the treatments.

border zone and the center of the infarct of the miR-29B-treated hearts ( $p=0.19$ and $p=0.09$, respectively, Fig. 5D).

\section{Raman microspectroscopy reveals significantly altered ECM-related peaks at the border zone of the infarcted myocardium}

To reliably detect subtle changes in ECM maturity and orientation, we used Raman microspectroscopy to obtain a biomolecular fingerprint to gain more information regarding the ECM at the border zone of miR-29B-treated infarcts with respect to the presence and quality of specific ECM components and their relative abundancies. Raman microspectroscopy has been used to distinguish various stages of cardiomyogenic differentiation, ${ }^{22}$ as well as detecting changes in ECM constituents after remodeling. ${ }^{23}$ Thus, by comparing between samples, changes in the ECM can be revealed. Within this study, we detected significant differences in the Raman spectra at distinct regions of the infarcted myocardium, namely the center of the infarct and the border zone (Fig. 6 and Sup- plementary Fig. S3). Interestingly, a greater number of spectra were significantly different at the border zone, which is where the miR-loaded hydrogel solutions were injected at the point at which infarction was induced. A large proportion of these reduced spectral peaks can be attributed to collagen $^{24}$ (Table 2), whereby the signals of these peaks were significantly decreased with the treatment of miR-29B compared with the control of miR-239B. In addition, the peaks of desmosine/isodesmosine, which are elastin crosslinking amino acids, ${ }^{25}$ were significantly reduced in the miR29B-treated border zones compared with the miR-239B controls, which is supportive of the quantification of elastin expression (Fig. 5A, B).

\section{Discussion}

Adverse ECM remodeling following MI remains still a considerable contributor toward end-stage heart failure. ${ }^{2}$ This study demonstrates the effectiveness of delivering exogenous miR-29B to the infarcted myocardium to affect localized

Table 2. Significantly Different Peaks Detected Between miR-239B (Control) and miR-29B AT THE CEnTER AND THE Border Zone of THE Infarct

\begin{tabular}{|c|c|c|}
\hline Center of infarct & Border zone of infarct & Peaks in literature assignment \\
\hline $452,453,455$ & - & 454: ring torsion of phenylalanine \\
\hline 522 & 525 & 524: S-S disulfide stretching band of collagen \\
\hline - & 763 & 760: ring breathing tryptophan (proteins) \\
\hline 860 & $858,859,860$ & 859: tyrosine of collagen \\
\hline 862,863 & $862,863,865,866$ & 820-930: $\mathrm{C}-\mathrm{C}$ stretch of proline and hydroxyproline \\
\hline - & 881 & 884: proteins, including collagen I \\
\hline - & $935,937,938,940,941,942$ & 928-940: C-C vibrations of proline and backbone \\
\hline - & $948,950,953$ & 950-951: $\mathrm{CH}_{3}$ deformation in proteins ( $\alpha$-helix) \\
\hline - & 964 & 963: $\mathrm{CH}$ bending in proteins \\
\hline - & 967 & 966: desmosine/isodesmosine \\
\hline - & 1127 & 1128: $\mathrm{C}-\mathrm{N}$ stretching \\
\hline- & 1155 & 1155: $\mathrm{C}-\mathrm{C}, \mathrm{C}-\mathrm{N}$ stretching in proteins \\
\hline 1305 & - & 1302-1314: $\mathrm{CH}_{2}$ twisting and wagging in collagen \\
\hline- & 1506,1508 & 1506: $\mathrm{N}=\mathrm{H}$ bending \\
\hline 1518,1521 & - & 1518-1520: $\mathrm{C}=\mathrm{C}$ stretching \\
\hline 1573 & - & 1573: tryptophan \\
\hline 1580 & - & 1580: $\mathrm{C}-\mathrm{C}$ stretching \\
\hline 1600,1601 & - & 1600: amide I band of proteins due to $\mathrm{C}=\mathrm{O}$ stretching \\
\hline 1610 & - & 1610: $\mathrm{NH}_{2}$ \\
\hline 1737 & - & 1736: $\mathrm{C}=\mathrm{O}$ \\
\hline
\end{tabular}

Detected peaks were assigned according to previously published reports. ${ }^{30}$ 
delivery and address adverse myocardial remodeling by harnessing endogenous molecules. ${ }^{5}$ In previous studies, exogenous miR-29B has been demonstrated to reduce fibrosis in other settings, ${ }^{12,26-28}$ yet this is the first reported demonstration in an MI model and specifically using a localized biomaterial delivery system (in this case a hyaluronan-based hydrogel). Although we found significant cardiac functional improvements following the administration of miR-29B using an injectable hyaluronic acid hydrogel, we did not identify the significant difference in the size of the ventricular scar between the miR-29B treatment and miR-239B controls anticipated. However, it should be reiterated that this proof-ofprinciple study has a low number of animals and therefore expanding this into a full investigation with a larger number of animals could reveal a statistically significant effect of the miR-29B treatment on ECM deposition (particularly collagen type I) and other parameters.

It is accepted that the constituents of the scar play a significant role in the compliance of scar tissue and contribution toward functional support following MI. ${ }^{29}$ Therefore, we sought to seek out more information regarding the components of the scar ECM. Although immunostaining did not reveal the significant difference in the collagen content within the center of the scar or at the border zone of the scar or at the remote myocardium that was anticipated (Fig. 4), polarized light microscopy revealed a significantly reduced presence of green immature fibers at the border zone of hearts treated with miR-29B compared with hearts treated with miR-239B. This corresponds to a significantly increased presence of red/ orange, more mature collagen fibers at the border zone of miR29B-treated infarcts. These mature collagen fibers are stronger and were aligned circumferentially with the myocardial wall, which gives compensatory support to the infarcted myocardium. Biomaterial scaffolds are capable of having a spatiotemporal release of therapeutics that is responsive to the biomaterial diffusion properties and interaction with a supporting host environment. ${ }^{6,23}$ The same applies in this study whereby we hypothesize that following MI and hydrogel injection, the overlapping phases of repair and tissue remodeling do occur within the infarcted myocardium leading to the synthesis of newly deposited ECM. However, later on, during hydrogel degradation and subsequent release of the therapeutic miR, additional collagen fiber formation and maturation is modulated, and most potently, at the border zone of the infarct, where the hydrogel had been delivered (Fig. 1E). The Raman microspectroscopy data were sufficiently sensitive toward detecting subtle changes in ECM orientation and maturity, whereby a more profound number of spectral peaks were significantly different in the border zone than the center of the infarct when comparing miR239B- and miR-29B-treated hearts ( 25 vs. 16 peaks, Fig. 6 , Supplementary Fig. S3 and Table 2) ${ }^{30}$ In addition, elastin production was significantly reduced at the border zone of infarcts treated with miR-29B compared to those treated with $\mathrm{miR}-239 \mathrm{~B}$ (Fig. $5 \mathrm{~A}-\mathrm{C}, 2.983 \pm 0.6764$ vs. $4.396 \pm$ $0.3207 \mathrm{GVI} / \mathrm{mm}^{2}$ ). This is important as it is widely established that miR-29B inhibits translation of the elastin gene $E L N .^{31}$ As elastin is known to be a pliable structure, and we detected more mature collagen fibers at the border zone of miR-29B-treated infarcts, we hypothesize that the border zones of the miR-29B-treated infarcts are stiffer than those of the miR-239B-treated infarcts.
The injection of biomaterial scaffolds does have an impact on myocardial remodeling and many different scaffolds have been injected into infarcted myocardium in previous studies, resulting in improvement in ventricular function due to ventricular thickening. ${ }^{32}$ Clinical trials with alginate as an injectable material treating MI have now been reported, ${ }^{33}$ yielding positive results in the first-in-man trials. ${ }^{34}$ The use of the hyaluronan-based hydrogel with no miR was considered in this study (Supplementary Fig. S1). Hydrogels with miR29B embedded still yielded a significant improvement in cardiac function compared with empty hydrogels, which signifies that this particular miR sequence is responsible for the improvements detected in cardiac function. Infarcts treated with hydrogels containing no miRs had a similar ejection fraction to infarcts treated with hydrogels containing the nontargeting miR-239B (Supplementary Fig. S1). In general, infarcts treated with only the hyaluronan-based hydrogel had a greater ejection fraction than infarcts treated with saline only, which demonstrates that in this instance, the injection of the hydrogel alone was itself, contributing a beneficial effect. This adds strength to the observations of this study such that injectable biomaterials are suitable for cardiac repair and are also suitable for delivering beneficial biomolecules such as miRs. The use of such a hydrogel reservoir of miR-29B is advantageous in achieving targeted delivery, but also a localized release of miR-29B. There are extensive reports of hyaluronic/PEG hydrogels whereby they have been used as carriers factor cells ${ }^{35,36}$ and nonviral gene therapeutics. ${ }^{35}$ For the most part, these investigations have been done in vitro, and the release profiles have been evaluated using PBS or hyaluronidase. In these studies, it was reported that burst release profiles can range from $20 \%$ to $65 \%$ in the first 2 days in the presence of PBS and burst release profiles ranged from $40 \%$ to $70 \%$ in the presence of hyaluronidase. Although the translation of in vitro release profiles to an in vivo situation is extremely difficult, we can assume that the miRs penetrate the infarct border zone tissue through the processes of hyaluronan degradation, PEG degradation, miR diffusion from the hydrogel, and the local tissue response.

The use of the hyaluronan-based hydrogel in combination with miR-29B in this study led to a tendency of increased blood vessel density at the border zone and center of the infarcts (Fig. 5D). It is important that the quantification of blood vessel density involved the identification of vessels containing both endothelial, CD31-expressing, and smooth muscle, $\alpha$-SMA-expressing, cells as the formation of single capillaries does not necessarily indicate an increase in blood flow, due to the ease of regression of vessels without smooth muscle. ${ }^{37}$ The injection of hyaluronic acid has been previously shown to increase angiogenesis in pathological settings such as $\mathrm{MI}^{38}$ and hind limb ischemia. ${ }^{39}$ Such reports are in agreement with the trends observed in this study (although not statistically significant) that the addition of miR-29B could have a positive influence on blood vessel formation due to its effect on ECM remodeling, which could be directly involved in the formation of blood vessels, or indirectly exerting positive biomechanical cues from the surrounding ECM.

Overall, the results of this proof-of-principle study provide robust evidence that miR-29B is a strong candidate toward modulating the aggressive synthesis of ECM that aims to compensate the infarcted myocardium. In addition, the use of an injectable hyaluronan-based system for a 
targeted delivery to elicit a regional effect was effective. Although strong trends were observed in histochemical analyses using the treatment of miR-29B (e.g., in the case of increased scar vascularity), statistically significant differences were not detected, potentially due to the low animal numbers used in this study. However, Raman microspectroscopy was sufficiently sensitive to detect significant differences in aspects of ECM maturation and structural orientation. Taking all this into account, this study gives strong evidence regarding the potential of this platform and provides a basis toward refinement of parameters to be tested in future evaluations of this platform.

\section{Conclusion}

In this study, we have shown that local hydrogel-based delivery of miR-29B to the infarcted myocardium at the border zone had a location-specific effect on ECM orientation and constituency. Collagen deposition was modulated, whereby the production of new immature collagen fibers at the border zone was reduced with a treatment of miR-29B. The deposition of elastin was significantly reduced at the border zone of the infarcted myocardium when miR-29B was delivered, and this group also had a strong tendency toward increased blood vessel density at both the border zone and center of the infarcted myocardium. Raman microspectroscopy was sufficiently sensitive to detect significant differences in aspects of ECM maturation and structural orientation, particularly with regard to the presence of elastin, which is supportive of the immunohistological elastin data, and discrete molecular changes within collagen fiber structure. Together, these promising proof-of-principle results demonstrate the feasibility of using injectable ECM-based biomaterials as local delivery reservoirs of therapeutics, and in this instance, the miR-29B therapy to modulate the remodeling of the ECM following MI.

\section{Acknowledgments}

This work was supported by funding from the European Union's Seventh Framework Program for research, technological development, and demonstration under grant agreement no. 331430 (to M.M.), as well as the Ministry of Science, Research and the Arts of Baden-Württemberg (33729.55-3/214 and SI-BW 01222-91). The authors thank Daniel Alejandro Cavajal Berrio (Department of Women's Health, University Tübingen, Germany) for his assistance in generating the graphical representations, Mariasole Zumbo (University of Modena and Reggio Emilia, Italy and Fraunhofer IGB Stuttgart, Germany) for assistance in preparing the immunohistological staining, and Carlos Molina (Fraunhofer IGB Stuttgart, Germany) for performing the Raman microspectroscopical measurements.

\section{Disclosure Statement}

No competing financial interests exist.

\section{References}

1. WHO Fact Sheet No. 310. Available at www.who.int/ mediacentre/factsheets/fs310/en/ (last accessed May 2014).

2. Steg, P.G., James, S.K., Atar, D., Badano, L.P., Lundqvist, C.B., Borger, M.A., Di Mario, C., Dickstein, K., Ducrocq,
G., Fernandez-Aviles, F., Gershlick, A.H., Giannuzzi, P., Halvorsen, S., Huber, K., Juni, P., Kastrati, A., Knuuti, J., Lenzen, M.J., Mahaffey, K.W., Valgimigli, M., van't Hof, A., Widimsky, P., Zahger, D., Bax, J.J., Baumgartner, H., Ceconi, C., Dean, V., Deaton, C., Fagard, R., FunckBrentano, C., Hasdai, D., Hoes, A., Kirchhof, P., Knuuti, J., Kolh, P., McDonagh, T., Moulin, C., Popescu, B.A., Reiner, Ž., Sechtem, U., Sirnes, P.A., Tendera, M., Torbicki, A., Vahanian, A., Windecker, S., Hasdai, D., Astin, F., ÅströmOlsson, K., Budaj, A., Clemmensen, P., Collet, J.-P., Fox, K.A., Fuat, A., Gustiene, O., Hamm, C.W., Kala, P., Lancellotti, P., Maggioni, A.P., Merkely, B., Neumann, F.-J., Piepoli, M.F., Van de Werf, F., Verheugt, F., and Wallentin, $\mathrm{L}$; The Task Force on the management of ST-segment elevation acute myocardial infarction of the European Society of Cardiology (ESC). ESC Guidelines for the management of acute myocardial infarction in patients presenting with ST-segment elevation. Eur Heart J 33, 2569, 2012.

3. Berk, B.C., Fujiwara, K., and Lehoux, S. ECM remodeling in hypertensive heart disease. J Clin Invest 117, 568, 2007.

4. Sun, Y., Cleutjens, J.P., Diaz-Arias, A.A., and Weber, K.T. Cardiac angiotensin converting enzyme and myocardial fibrosis in the rat. Cardiovasc Res 28, 1423, 1994.

5. Monaghan, M., Greiser, U., Wall, J.G., O'Brien, T., and Pandit, A. Interference: an alteRNAtive therapy following acute myocardial infarction. Trends Pharmacol Sci 33, 635, 2012.

6. Monaghan, M., and Pandit, A. RNA interference therapy via functionalized scaffolds. Adv Drug Deliv Rev 63, 197, 2011.

7. Li, Z., Hassan, M.Q., Jafferji, M., Aqeilan, R.I., Garzon, R., Croce, C.M., van Wijnen, A.J., Stein, J.L., Stein, G.S., and Lian, J.B. Biological functions of miR-29b contribute to positive regulation of osteoblast differentiation. J Biol Chem 284, 15676, 2009.

8. Roderburg, C., Urban, G.-W., Bettermann, K., Vucur, M., Zimmermann, H., Schmidt, S., Janssen, J., Koppe, C., Knolle, P., Castoldi, M., Tacke, F., Trautwein, C., and Luedde, T. Micro-RNA profiling reveals a role for miR-29 in human and murine liver fibrosis. Hepatology 53, 209, 2011.

9. van Rooij, E., Sutherland, L.B., Thatcher, J.E., DiMaio, J.M., Naseem, R.H., Marshall, W.S., Hill, J.A., and Olson, E.N. Dysregulation of microRNAs after myocardial infarction reveals a role of miR-29 in cardiac fibrosis. Proc Nat Acad Sci U S A 105, 13027, 2008.

10. Monaghan, M., Greiser, U., Cao, H., Wang, W., and Pandit, A. An antibody fragment functionalized dendritic PEGylated poly(2-(dimethylamino)ethyl diacrylate) as a vehicle of exogenous microRNA. Drug Deliv Transl Res 2, 406, 2012.

11. Liu, Y., Taylor, N.E., Lu, L., Usa, K., Cowley, A.W., Jr., Ferreri, N.R., Yeo, N.C., and Liang, M. Renal medullary microRNAs in Dahl salt-sensitive rats: miR-29b regulates several collagens and related genes. Hypertension 55, 974, 2010.

12. Monaghan, M., Browne, S., Schenke-Layland, K., and Pandit, A. A collagen-based scaffold delivering exogenous microrna-29B to modulate extracellular matrix remodeling. Mol Ther 22, 786, 2014.

13. Mencia Castano, I., Curtin, C.M., Shaw, G., Murphy, J.M., Duffy, G.P., and O'Brien, F.J. A novel collagennanohydroxyapatite microRNA-activated scaffold for tissue engineering applications capable of efficient delivery of both miR-mimics and antagomiRs to human mesenchymal stem cells. J Control Release 200, 42, 2015.

14. Duan, J., Gherghe, C., Liu, D., Hamlett, E., Srikantha, L., Rodgers, L., Regan, J.N., Rojas, M., Willis, M., Leask, A., 
Majesky, M., and Deb, A. Wnt1/betacatenin injury response activates the epicardium and cardiac fibroblasts to promote cardiac repair. EMBO J 31, 429, 2012.

15. Monaghan, M.G., Linneweh, M., Liebscher, S., Van Handel, B., Layland, S.L., and Schenke-Layland, K. Endocardial-tomesenchymal transformation and mesenchymal cell colonization at the onset of human cardiac valve development. Development 143, 473, 2016.

16. Djokic, J., Fagotto-Kaufmann, C., Bartels, R., Nelea, V., and Reinhardt, D.P. Fibulin-3, -4 , and -5 are highly susceptible to proteolysis, interact with cells and heparin, and form multimers. J Biol Chem 288, 22821, 2013.

17. York, T., Kahan, L., Lake, S.P., and Gruev, V. Real-time high-resolution measurement of collagen alignment in dynamically loaded soft tissue. J Biomed Opt 19, 066011, 2014.

18. Pudlas, M., Berrio, D.A.C., Votteler, M., Koch, S., Thude, S., Walles, H., and Schenke-Layland, K. Non-contact discrimination of human bone marrow-derived mesenchymal stem cells and fibroblasts using Raman spectroscopy. Med Laser Appl 26, 119, 2011.

19. Wolman, M., and Kasten, F.H. Polarized light microscopy in the study of the molecular structure of collagen and reticulin. Histochemistry 85, 41, 1986.

20. Yu, S.Y., Tozzi, C.A., Babiarz, J., and Leppert, P.C. Collagen changes in rat cervix in pregnancy-polarized light microscopic and electron microscopic studies. Proc Soc Exp Biol Med 209, 360, 1995.

21. Hirshberg, A., Sherman, S., Buchner, A., and Dayan, D. Collagen fibres in the wall of odontogenic keratocysts: a study with picrosirius red and polarizing microscopy. J Oral Pathol Med 28, 410, 1999.

22. Brauchle, E., Knopf, A., Bauer, H., Shen, N., Linder, S., Monaghan, Michael G., Ellwanger, K., Layland, Shannon L., Brucker, Sara Y., Nsair, A., and Schenke-Layland, K. Non-invasive chamber-specific identification of cardiomyocytes in differentiating pluripotent stem cells. Stem Cell Rep 6, 188, 2016.

23. Browne, S., Monaghan, M.G., Brauchle, E., Berrio, D.C., Chantepie, S., Papy-Garcia, D., Schenke-Layland, K., and Pandit, A. Modulation of inflammation and angiogenesis and changes in ECM GAG-activity via dual delivery of nucleic acids. Biomaterials 69, 133, 2015.

24. Votteler, M., Carvajal Berrio, D.A., Pudlas, M., Walles, H., Stock, U.A., and Schenke-Layland, K. Raman spectroscopy for the non-contact and non-destructive monitoring of collagen damage within tissues. J Biophotonics 5, 47, 2012.

25. Frushour, B.G., and Koenig, J.L. Raman scattering of collagen, gelatin, and elastin. Biopolymers 14, 379, 1975.

26. Maegdefessel, L., Azuma, J., Toh, R., Merk, D.R., Deng, A., Chin, J.T., Raaz, U., Schoelmerich, A.M., Raiesdana, A., Leeper, N.J., McConnell, M.V., Dalman, R.L., Spin, J.M., and Tsao, P.S. Inhibition of microRNA-29b reduces murine abdominal aortic aneurysm development. J Clin Invest 122, 497, 2012.

27. Xiao, J., Meng, X.-M., Huang, X.R., Chung, A.C.K., Feng, Y.-L., Hui, D.S.C., Yu, C.-M., Sung, J.J.Y., and Lan, H.Y. miR-29 inhibits bleomycin-induced pulmonary fibrosis in mice. Mol Ther 20, 1251, 2012.

28. Zhang, Y., Huang, X.-R., Wei, L.-H., Chung, A.C.K., Yu, C.-M., and Lan, H.-Y. miR-29b as a therapeutic agent for angiotensin II-induced cardiac fibrosis by targeting TGF[beta]/Smad3 signaling. Mol Ther 22, 974, 2014.

29. Holmes, J.W., Nunez, J.A., and Covell, J.W. Functional implications of myocardial scar structure. Am J Physiol Heart Circ Physiol 272, H2123, 1997.

30. Movasaghi, Z., Rehman, S., and Rehman, I.U. Raman spectroscopy of biological tissues. Appl Spectrosc Rev 42, 493, 2007.

31. Zhang, P., Huang, A., Ferruzzi, J., Mecham, R.P., Starcher, B.C., Tellides, G., Humphrey, J.D., Giordano, F.J., Niklason, L.E., and Sessa, W.C. Inhibition of microRNA 29 enhances elastin levels in cells haploinsufficient for elastin and in bioengineered vessels. Arterioscler Thromb Vasc Biol 32, 756, 2012.

32. Ungerleider, J.L., and Christman, K.L. Concise review: injectable biomaterials for the treatment of myocardial infarction and peripheral artery disease: translational challenges and progress. Stem Cells Transl Med 3, 1090, 2014.

33. Inc., I.H. IK-5001 for the prevention and remodling of the ventricle and congestive heart failure after acute myocardial infarction. NCT01226563, 2010

34. Frey, N., Linke, A., Suselbeck, T., Muller-Ehmsen, J., Vermeersch, P., Schoors, D., Rosenberg, M., Bea, F., Tuvia, S., and Leor, J. Intracoronary delivery of injectable bioabsorbable scaffold (IK-5001) to treat left ventricular remodeling after ST-elevation myocardial infarction: a first-in-man study. Circ Cardiovasc Interv 7, 806, 2014.

35. Wieland, J.A., Houchin-Ray, T.L., and Shea, L.D. Nonviral vector delivery from PEG-hyaluronic acid hydrogels. J Control Release 120, 233, 2007.

36. Jeong, C.G., Francisco, A.T., Niu, Z., Mancino, R.L., Craig, S.L., and Setton, L.A. Screening of hyaluronic acidpoly(ethylene glycol) composite hydrogels to support intervertebral disc cell biosynthesis using artificial neural network analysis. Acta Biomater 10, 3421, 2014.

37. Benjamin, L.E., Hemo, I., and Keshet, E. A plasticity window for blood vessel remodelling is defined by pericyte coverage of the preformed endothelial network and is regulated by PDGF-B and VEGF. Development 125, 1591, 1998.

38. Chen, C.H., Wang, S.S., Wei, E.I., Chu, T.Y., and Hsieh, P.C. Hyaluronan enhances bone marrow cell therapy for myocardial repair after infarction. Mol Ther 21, 670, 2013.

39. Tang, Z.C.W., Liao, W.-Y., Tang, A.C.L., Tsai, S.-J., and Hsieh, P.C.H. The enhancement of endothelial cell therapy for angiogenesis in hindlimb ischemia using hyaluronan. Biomaterials 32, 75, 2011.

Address correspondence to: Katja Schenke-Layland, PhD, MSc Department of Cell and Tissue Engineering Fraunhofer IGB Stuttgart Nobelstrasse 12 Stuttgart 70569 Germany

E-mail: katja.schenke-layland@igb.fraunhofer.de

Received: December 2, 2016 Accepted: April 3, 2017 Online Publication Date: May 22, 2017 